\title{
Hidden Markov Process Based Dynamic Spectrum Access for Cognitive Radio
}

\author{
Thao Nguyen ${ }^{\dagger, *}$, Brian L. Mark*, Yariv Ephraim* \\ ${ }^{\dagger}$ Shared Spectrum Company \\ 1595 Spring Hill Rd, Suite 110 \\ Vienna, VA 22182 \\ *Dept. of Electrical and Computer Engineering \\ George Mason University, MS 1G5 \\ 4400 University Drive, Fairfax, VA \\ email: tnguyed@gmu.edu, bmark@gmu.edu, yephraim@gmu.edu
}

\begin{abstract}
Cognitive radio is an emerging technology for sensing and dynamic access of spectrum in mobile radio environments. It aims at dynamically allocating unused bandwidth among secondary users without causing harmful interference to the primary users. This approach, which has clear economical benefits, has recently attracted significant research effort. In this paper, we propose a new approach to dynamic spectrum access in which the occupancy state of each frequency band at each time instant is estimated, and available bands are allocated accordingly. Estimation is performed from power spectral density measurements which are assumed to obey a hidden Markov process. The value of the hidden state represents the status of a given frequency band which could be free or occupied. We have trained the system using real spectrum measurements, and tested it on simulated data for which the occupancy state of each frequency band at each time instant is known. We demonstrate the performance of the proposed approach and compare it with a simple energy detector which has previously been proposed for this application. ${ }^{1}$
\end{abstract}

Index Terms-Cognitive radio, hidden Markov process, dynamic spectrum access

\section{INTRODUCTION}

It is well known that statically allocated radio bandwidth remains idle for extended periods of time, see, e.g., [1]. To better utilize the bandwidth, and thus accommodate an ever increasing demand, cognitive radio (CR) technologies have been proposed to allow secondary (unlicensed) users to make use of idle spectrum without causing harmful interference to primary (licensed) users. In the jargon of CR, idle frequency bands are referred to as white spaces. A key feature of a CR is its ability to sense the radio environment and dynamically switch transmission from one channel to another.

Sensing the environment is usually done by measuring the power spectral density and estimating the status of the primary user as being active or idle. This formulation is amenable to estimating a state variable from some given noisy and possibly incomplete observations. Indeed, several authors have formulated the problem as that of estimating the state of a

\footnotetext{
${ }^{1}$ This work was supported in part by the U.S. National Science Foundation under Grant CCF-0916568.
}

hidden Markov process (HMP). A survey of hidden Markov processes may be found in [2]. In [3], real-time measurements collected in the paging band $928-948 \mathrm{MHz}$ were used to validate the on-off behavior of primary users. In [4], [5], a spectrum sensing approach based on an HMP and quickest detection was proposed. In [6], [7], an HMP-based classifier is used in conjunction with a cyclostationary detector for dynamic spectrum access. All of these approaches rely on finite-alphabet HMPs, and only [3] employs real spectrum occupancy measurements.

In this paper, we pursue hidden Markov modeling of the cognitive radio problem. In its general form, it is the problem of sensing broadband signals, representing spectrum from several users, and deciding activity level for each sub-band that was initially assigned to a particular licensed user. Thus, a multi-state HMP whose parameter is recursively adapted, is required. Here we focus on a subset of this problem in which there is a single primary user and we assume that the environment is stationary. For this narrowband cognitive radio problem, we assume a two-state HMP with continuous alphabet measurements. One state represents activity of the primary user while the other represents idleness of the user. The parameter of the HMP is estimated offline from real measurements using the Baum algorithm. Given this parameter, the state of activity of the primary user at a given time and frequency band is determined using the forwardbackward algorithm. Thus, at each time instant, the posterior probability of each of the two states is estimated, and a maximum a-posteriori decision is implemented. Contrary to earlier attempts, our approach does not require quantization of the spectral measurements which may inevitably obscure the goals of CR. Furthermore, we recursively predict the activity of the primary user from a derivative of the forward recursion.

We have carefully implemented and tested our HMP-based CR system using real spectrum occupancy measurements collected in the 380-382 MHz band over a period of 25 hours. Our system outperforms a commonly used energy detector especially at low signal-to-noise ratios. While the energy 


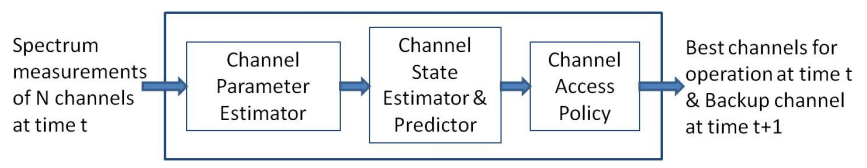

Fig. 1. System model for HMP-based spectrum access model in CR.

detector implements a likelihood ratio for Gaussian densities, the HMP based approach effectively implements a likelihood ratio for mixtures of Gaussian densities, and consecutive tests are not independent due to the inherit memory of the HMP.

The remainder of the paper is organized as follows. Section II develops the proposed HMP-based system. Section III discusses the system performance evaluation using real spectrum measurement data. Concluding remarks are given in Section IV.

\section{System ModeL}

The configuration of the proposed HMP-based system for dynamic spectrum access is shown in Fig. 1. Time is assumed to be partitioned into slots of duration $\Delta$. Thus, we assume a discrete-time model, where the time variable $t$ takes values in $\{0,1, \ldots\}$. The radio spectrum of interest consists of $N$ channels. The input to the system at time $t$ consists of an estimate of the power spectral density in units of dB. Each channel is modeled as a two-state HMP with conditionally Gaussian observations. The output of the system consists of a list of available channels for CR operation at time $t$ and a corresponding list of backup channels for time $t+1$.

The system consists of three main components: channel parameter estimator, channel state estimator and predictor, and channel access policy. The channel parameter estimator estimates the HMP parameter for a given channel from spectrum measurements collected offline as training data. The channel state estimator and predictor uses the estimated HMP parameter for a given channel to estimate the current state (at time $t$ ) and to predict the next state (at time $t+1$ ) of each channel. Finally, the channel access policy identifies a best channel for CR operation at time $t$ and a list of backup channels for $\mathrm{CR}$ operation at time $t+1$ in case the best channel at time $t$ becomes busy. The number of backup channels to be identified by the channel access policy may be configurable.

\section{A. Channel parameter estimator}

The channel parameter estimator estimates an HMP parameter associated with each of the $N$ channels based on offline training data. For a given channel, the HMP parameter can be estimated using the Baum re-estimation algorithm [2], [8].

An HMP may be viewed as a discrete-time bivariate random process $\left\{\left(S_{t}, Y_{t}\right), t=1,2, \ldots\right\}$, where $\left\{S_{t}\right\}$ is the hidden process and $\left\{Y_{t}\right\}$ is the observable process. The hidden process $\left\{S_{t}\right\}$ is a discrete-time finite state homogeneous Markov chain. The random variables $\left\{Y_{t}\right\}$ are conditionally independent given $\left\{S_{t}\right\}$. Furthermore, the distribution of $Y_{t}$ is timeinvariant and depends on $\left\{S_{t}\right\}$ only through $S_{t}$.
The hidden process $\left\{S_{t}\right\}$ takes values in a finite set $\mathcal{S}=$ $\{0, \ldots, M-1\}$. In this paper, we shall focus on the case $M=2$, such that state 1 represents that the primary user is on, whereas state 0 represents a white space on the channel at the current time $t$. Our approach can easily be generalized to incorporate $m$ steps of dependency or memory such that each state represents a sequence of on/off states of the primary user at times $t-m+1, t-m+2, \ldots, t$. In this case, the number of states $M=2^{m}$, resulting in a higher-order HMP (cf. [5]), i.e., an HMP of order $m$.

For each $t$, the observation $Y_{t}$ takes values in an observation space $\mathcal{Y}$. We assume in general that the observation is represented by a vector consisting of $K$ components. If the observation represents the total power of the channel we have $K=1$. If the channel is divided into $K>1$ frequency bins within its bandwidth, the observation consists of $K$ components such that each component represents the total power in a frequency bin. Thus, in general we have $\mathcal{Y}=\mathbb{R}^{K}$, when power is measured in units of $\mathrm{dBm}$. An HMP with $m>1$ and $K>1$ may capture existing temporal and spectral cross-correlation and thus allow better sensing of the channel.

Let $\pi_{i}=P\left(S_{1}=i\right), i \in \mathcal{S}$, denote the probability that the initial state is $i$. Let $\pi=\left\{\pi_{i}, i \in \mathcal{S}\right\}$ denote a vector representing the initial probability distribution of $\left\{S_{t}\right\}$. Let $A=\left\{a_{i j}, i, j \in \mathcal{S}\right\}$ denote the transition matrix of $\left\{S_{t}\right\}$. Let $b(y \mid i)$ denote the probability density function of the observation vector $y$ given the state $i$. We assume that $b(y \mid i)$ is given by a multivariate Gaussian density with mean vector $\mu_{i}$ and covariance matrix $\Sigma_{i}$ :

$$
b(y \mid i) \sim \mathcal{N}\left(\mu_{i}, \Sigma_{i}\right)
$$

Relying on asymptotic properties of the log-power spectral density estimate [9], we assume that each $\Sigma_{i}$ is a diagonal matrix. Let $\mu=\left\{\mu_{i}, i \in \mathcal{S}\right\}$ and $\Sigma=\left\{\Sigma_{i}, i \in \mathcal{S}\right\}$. The HMP for a given channel is then characterized by the parameter $\lambda=(\pi, A, \mu, \Sigma)$.

Let $s^{T}=\left\{s_{t}, t=1, \ldots, T, s_{t} \in \mathcal{S}\right\}$ denote the sequence of hidden states of the HMP from time 1 to time $T$. The probability of the state sequence $s^{T}$ is given by

$$
p_{\lambda}\left(s^{T}\right)=\prod_{t=1}^{T} a_{s_{t-1} s_{t}},
$$

where we have used the convention $a_{s_{0} s_{1}}=\pi_{s_{1}}$. Let $y^{T}=\left\{y_{t}, t=1, \ldots, T, y_{t} \in \mathcal{Y}\right\}$, denote the sequence of observations from time 1 to time $T$.

We denote the probability density function of $y$ under the HMP parameter $\lambda$ by $p_{\lambda}(y)$ and the conditional density of $y$ given the hidden state sequence $s$ by $p_{\lambda}(y \mid s)$. The conditional density of $y^{T}$ given $s^{T}$ is given by

$$
p_{\lambda}\left(y^{T} \mid s^{T}\right)=\prod_{t=1}^{T} b\left(y_{t} \mid s_{t}\right)
$$


The density of $y^{T}$ can be expressed as

$$
p_{\lambda}\left(y^{T}\right)=\sum_{s^{T}} p_{\lambda}\left(s^{T}, y^{T}\right)=\sum_{s^{T}} \prod_{t=1}^{T} a_{s_{t-1} s_{t}} b\left(y_{t} \mid s_{t}\right) .
$$

An HMP parameter estimate $\hat{\lambda}$ is obtained by maximizing the likelihood given an observation sequence $y^{T}$ :

$$
\hat{\lambda}=\arg \max _{\lambda} \ln p_{\lambda}\left(y^{T}\right)=\arg \max _{\lambda} \ln \sum_{s^{T}} p_{\lambda}\left(s^{T}, y^{T}\right),
$$

where the maximization is performed by the Baum reestimation algorithm. This algorithm generates a sequence of HMP parameter estimates with nondecreasing likelihood values. Each iteration of the Baum algorithm starts with an the current parameter $\lambda_{\iota}$ and estimates a new parameter $\lambda_{\iota+1}$ by maximizing the following auxiliary function

$$
\phi\left(\lambda_{\iota+1}\right)=\sum_{s^{T}} p_{\lambda_{\iota}}\left(s^{T} \mid y^{T}\right) \ln p_{\lambda_{\iota+1}}\left(s^{T}, y^{T}\right)
$$

over the HMP parameter $\lambda_{\iota+1}$. The algorithm is terminated when a convergence criterion is satisfied, e.g., when the relative difference of the $\log$-likelihoods $\ln p_{\lambda_{\iota+1}}\left(y^{T}\right)$ and $\ln p_{\lambda_{\iota}}\left(y^{T}\right)$ is smaller than or equal to a given threshold.

Let $q_{t}(i, j)=P\left(S_{t-1}=i, S_{t}=j \mid y^{T}\right)$ and $q_{t}(j)=$ $P\left(S_{t}=j \mid y^{T}\right)$ under the HMP parameter $\lambda$. Maximization of the auxiliary function in (6) results in the following reestimation formulas:

$$
\begin{aligned}
\hat{\pi}_{i} & =q_{0}(i), \\
\hat{a}_{i j} & =\frac{\sum_{t=1}^{T} q_{t}(i, j)}{\sum_{j \in \mathcal{S}} \sum_{t=1}^{T} q_{t}(i, j)},
\end{aligned}
$$

and the parameters of the density $b\left(y_{t} \mid s_{t}=i\right) \sim \mathcal{N}\left(\mu_{i}, \Sigma_{i}\right)$ can be estimated as

$$
\begin{aligned}
\hat{\mu}_{i} & =\frac{\sum_{t=1}^{T} q_{t}(i) y_{t}}{\sum_{t=1}^{T} q_{t}(i)}, \\
\hat{\Sigma}_{i} & =\frac{\sum_{t=1}^{T} q_{t}(i)\left[I \odot\left(y_{t}-\hat{\mu}_{i}\right)\left(y-\hat{\mu}_{i}\right)^{\prime}\right]}{\sum_{t=1}^{T} q_{t}(i)},
\end{aligned}
$$

where $I$ denotes the identity matrix, $\odot$ denotes element-byelement matrix multiplication, and ' denotes matrix transpose. In (10), the operation involving $I$ and $\odot$ ensures that $\hat{\Sigma}_{i}$ is a diagonal matrix.

The probabilities $q_{t}(i, j)$ and $q_{t}(j)$ can be efficiently calculated using the forward-backward formulas as follows:

$$
\begin{aligned}
q_{t}(i, j) & =\frac{F_{t-1}(i) B_{t}(j) a_{i j} b\left(y_{t} \mid j\right)}{\sum_{i, j \in \mathcal{S}} F_{t-1}(i) B_{t}(j) a_{i j} b\left(y_{t} \mid j\right)}, 2 \leq t \leq T, \\
q_{t}(j) & =\frac{F_{t}(j) B_{t}(j)}{\sum_{i \in \mathcal{S}} F_{t}(i) B_{t}(i)}, 1 \leq t \leq T
\end{aligned}
$$

where $F_{t}(i)$ is the forward parameter for state $i$ at time $t$, and $B_{t}(j)$ is the backward parameter for state $j$ at time $t$. These parameters are given by

$$
\begin{aligned}
& F_{1}(i)=\pi_{i} b\left(y_{1} \mid i\right), \\
& F_{t}(i)=\sum_{j \in \mathcal{S}} F_{t-1}(j) a_{j i} b\left(y_{t} \mid i\right), 2 \leq t \leq T,
\end{aligned}
$$

and

$$
\begin{aligned}
B_{T}(j) & =1, \\
B_{t}(j) & =\sum_{i \in \mathcal{S}} B_{t+1}(i) a_{j i} b\left(y_{t+1} \mid i\right), 1 \leq t \leq T-1 .
\end{aligned}
$$

A proper scaling of the forward and backward probabilities is required. In particular, a recursive scaling is introduced, in which both $F_{t}(i)$ and $B_{t}(j)$ are normalized by $\sum_{i \in \mathcal{S}} F_{t}(i)$ at each time $t$. In addition, the calculation of each summand of the forward-backward formulas and of the probabilities $q_{t}(i, j)$ is done in the log domain due to the relatively small numbers involved. The values of $\ln b\left(y_{t} \mid s_{t}\right)$ are also shifted into the dynamic range of the computer prior to their summation, simply by replacing the out of range values with the min and max values of the dynamic range.

\section{B. Channel state estimator and predictor}

Spectrum sensing is one of the important features of a CR. The goal of the spectrum sensing is to estimate the true state of the channel, given measurements, with low probability of false alarm while maintaining high probability of detection. In most of the current spectrum sensing approaches, the channel state is estimated based only on the current measurement, not on the measurement history. With HMP-based spectrum sensing, we can utilize the estimated HMP parameter, which is computed based on the measurement history, beside the current measurement to estimate the current channel state more accurately. In addition, this approach provides the capability to predict the next channel state, whereas most of the current spectrum sensing approaches are not capable of doing so.

We present the filtering and prediction recursions in the rest of this section. Let $\hat{\lambda}=(\hat{\pi}, \hat{A}, \hat{\mu}, \hat{\Sigma})$ denote the current estimate of the HMP parameter for the channel. The prediction and filtering recursions are based on the conditional probabilities $p_{\hat{\lambda}}\left(s_{t} \mid y^{t-1}\right)$ and $p_{\hat{\lambda}}\left(s_{t} \mid y^{t}\right)$, respectively. The conditional probabilities $p\left(s_{t} \mid y^{t}\right)$ and $p\left(s_{t} \mid y^{t-1}\right)$ can be computed as follows [2]:

$$
p_{\hat{\lambda}}\left(s_{t} \mid y^{t}\right)=\frac{p_{\hat{\lambda}}\left(s_{t} \mid y^{t-1}\right) b\left(y_{t} \mid s_{t}\right)}{\sum_{s_{t}=1}^{M} p_{\hat{\lambda}}\left(s_{t} \mid y^{t-1}\right) b\left(y_{t} \mid s_{t}\right)}, 1 \leq t \leq T,
$$

where $p_{\hat{\lambda}}\left(s_{1} \mid y_{1}^{0}\right)=\hat{\pi}_{s_{1}}$ and

$$
p_{\hat{\lambda}}\left(s_{t} \mid y^{t-1}\right)=\sum_{s_{t-1} \in \mathcal{S}} \hat{a}_{s_{t-1} s_{t}} p_{\hat{\lambda}}\left(s_{t-1} \mid y^{t-1}\right), 2 \leq t \leq T .
$$

\section{Channel access policy}

The channel access policy makes decisions based on the sensing outcome from the channel state estimator and predictor. The goal is to prevent harmful interference to the primary user while maximizing the transmission time of the secondary user.

In dynamic spectrum access, a CR may begin data transmission on a given channel if the channel is sensed as idle. Conversely, if a CR is transmitting on a channel and senses the presence of a primary user, it must stop its data transmission 
in order to avoid causing harmful interference to the primary user. In practical hardware implementations of a CR, there exists a non-negligible latency between the time the channel is sensed and the time when the CR can begin or stop its data transmission on the channel, according as the channel is sensed as idle or busy at the current time [10], [11].

The channel access policy uses channel state estimation and prediction information to minimize the potential for interference between the primary user and the CR during this latency period. Let $t_{\text {lat }}$ denote the latency time required for a CR to stop or begin its data transmission after sensing the channel. The channel access policy allows the CR to transmit at the current time $t$ only if the current state estimate at time $t$ and the predicted state at time $t+t_{\text {lat }}$ indicate an idle channel. Likewise, the channel access policy forces an active CR to stop its data transmission if either the current state estimate or the predicted state at time $t+t_{\text {lat }}$ indicates a busy channel. The channel state estimate can be obtained using (17). Assuming that $t_{\text {lat }}$ is on the order of a slot time or smaller, the predicted state can be obtained using (18). For larger $t_{\text {lat }}$, a generalization of (18) to provide multi-step prediction could be used, at the expense of a loss in prediction accuracy and consequently, a more conservative channel access policy.

In a system with multiple channels, a CR may have a choice of several idle channels to use. In this case, the effective transmission time of the secondary user can be maximized by identifying a best channel and a list of backup channels. The criteria for selecting these channels would again be based on the channel state estimation and prediction information provided by (17) and (18). The best channel would be the one predicted to remain idle for the longest period of time starting from time $t$. Thus, the CR can minimize the frequency of switching among different channels during its data transmission. Backup channels would be those predicted to be idle at $t+1$. When the CR sensed that the current channel is no longer avaiable, it could quickly switch to one of the backup channels. Maintaining a list of backup channels can also help to reduce the rendezvous time needed to select a common idle channel for communications between a pair of CRs.

\section{Performance Analysis}

In this section, we evaluate the performance of the CR system discussed in Section II.

\section{A. Channel parameter estimation with real spectrum measure- ments}

We utilized the spectrum occupancy measurements collected on the rooftop of Shared Spectrum Company using a spectrum analyzer. Fig. 2 shows the occupancy spectrum measurements in 380-382 MHz band over 25 hours. The frequency resolution of the measurement is $10 \mathrm{kHz}$ and the repetition rate is 4.4 seconds. This spectrum band is occupied by primary users with narrow bandwidth emitters (i.e., $11 \mathrm{kHz}$ and $6 \mathrm{kHz}$ ). The plot shows that most of the channels are being used but not all channels have full duty cycle.

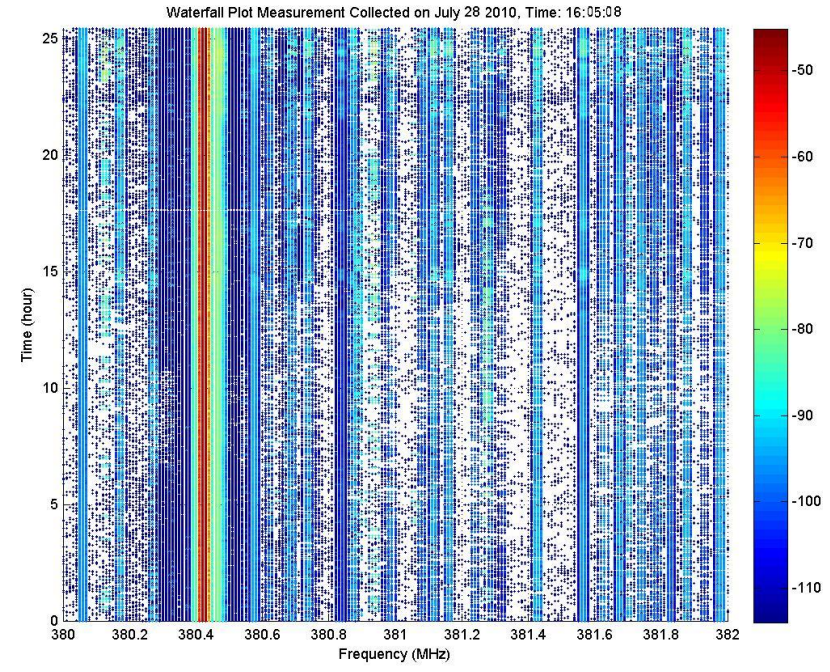

Fig. 2. Spectrum occupancy measurements in $380-382 \mathrm{MHz}$ range.
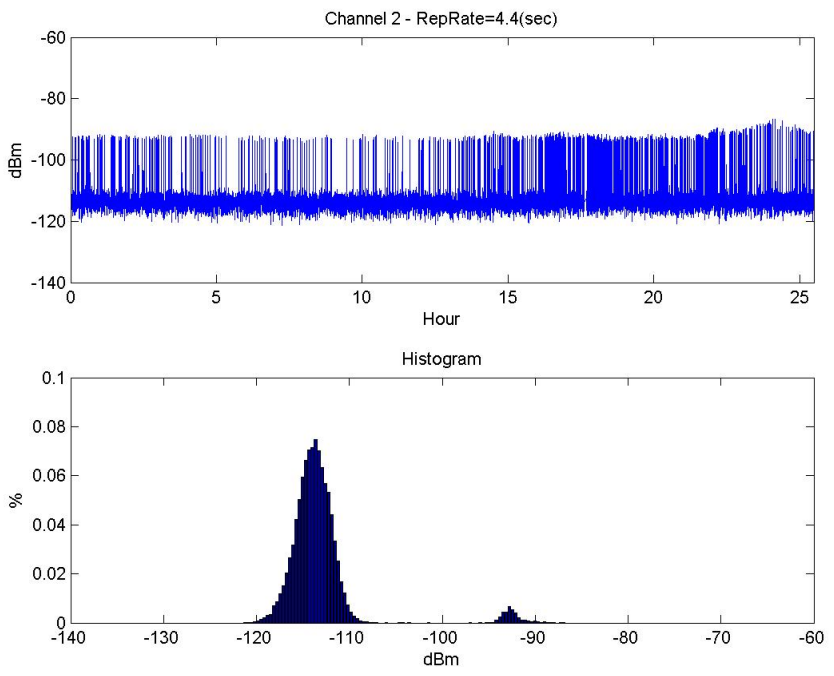

Fig. 3. Spectrum Measurement and Histogram Plots.

In this section, we only focus on HMP parameter estimation for a single channel and assume that there are two hidden states. We shall consider the channel with center frequency at $381.7375 \mathrm{MHz}$ and $11 \mathrm{kHz}$ bandwidth. The parameters of other channels in the band were estimated similarly. The number of observation symbols for this channel is 20860 . Fig. 3 shows the spectrum measurement and histogram plots of the channel. As shown in the figure, the transmissions on this channel have a high SNR but low duty cycle.

Given the measurements of the channel, we have used the algorithm described in Section II-A and estimated the HMP 
parameters as follows:

$$
\begin{aligned}
& \hat{\pi}=\left[\begin{array}{ll}
1 & 0
\end{array}\right], \\
& \hat{A}=\left[\begin{array}{ll}
0.9687 & 0.0313 \\
0.7970 & 0.2030
\end{array}\right], \\
& \hat{\mu}=\left\{\begin{array}{ll}
-113.9235, & -92.4308
\end{array}\right\}, \\
& \hat{\Sigma}=\left\{\begin{array}{ll}
3.4105, & 1.5993
\end{array}\right\} .
\end{aligned}
$$

The estimated parameter set shows consistency with measurement plots. The initial probability vector $\hat{\pi}$ has very high probability for the off state $\left(\hat{\pi}_{0}=1\right)$ and very low probability for the on state $\left(\hat{\pi}_{1}=0\right)$. The transition matrix $\hat{A}$ also indicates that the probability of the channel staying in the off state $\left(\hat{a}_{00}\right)$ is much higher than staying in the on state $\left(\hat{a}_{11}\right)$. The mean vector $\hat{\mu}$ shows that the mean value of the observations in the off state is equal to $-113.9 \mathrm{dBm}$ while that in the on state is $-92.4 \mathrm{dBm}$, which are very close to the measurements observed from the plot. Finally, the variance in the off state is about 3.4 while that in the on state is about 1.6 , which is again consistent with the plots.

\section{B. Channel state estimation and prediction}

To test our system, we have generated data from an HMP with the same parameter value as that estimated from the real data. For this simulated data, we have the observation sequence as well as the underlying hidden state sequence. This allows us to apply our sensing algorithm to the observation sequence, estimate the hidden state sequence, and compare it with the true state sequence. This procedure is necessary, since the state occupancy sequence for the real data is not known. More specifically, the following steps were carried out.

1) Generate a state sequence $\tilde{s}^{T}=\left\{\tilde{s}_{t}, t=1, \ldots, T\right\}$ using the estimated initial distribution $\hat{\pi}$ and transition matrix $\hat{A}$.

2) Generate an observation sequence $\tilde{y}^{T}=\left\{\tilde{y}_{t}, t=\right.$ $1, \ldots, T\}$ associated with the generated state sequence $\tilde{s}^{T}$ using the estimated mean vector $\hat{\mu}$ and standard deviation vector $\hat{\sigma}$.

3) Re-estimate the parameter set $\tilde{\lambda}$ of the channel from the observation sequence in Step 2). The new estimated parameter set $\tilde{\lambda}$ should be close to the parameter set $\hat{\lambda}$ used in Steps 1) and 2). We obtained

$$
\begin{aligned}
\tilde{\pi} & =\left[\begin{array}{ll}
1 & 0
\end{array}\right], \\
\tilde{A} & =\left[\begin{array}{ll}
0.9693 & 0.0307 \\
0.7990 & 0.2010
\end{array}\right], \\
\tilde{\mu} & =\left\{\begin{array}{ll}
-113.9428, & -92.4948
\end{array}\right\}, \\
\tilde{\Sigma} & =\left\{\begin{array}{ll}
4.9121, & 2.0220
\end{array}\right\} .
\end{aligned}
$$

4) Apply the filtering and prediction recursions using the new estimated parameter set $\tilde{\lambda}$ and the observation sequence $\tilde{y}^{T}$ to get the current state and predict the next state for every time $t$.

5) Compute the probability of false alarm, $P_{\text {fa }}$, and probability of detection, $P_{\mathrm{d}}$, by comparing the estimated state sequence in Step 4) with the state sequence $\tilde{s}^{T}$ generated

\begin{tabular}{|c|c|c|c|}
\hline SNR $(\mathrm{dB})$ & $P_{\mathrm{fa}}$ & $P_{\mathrm{d}}$ & $P_{\mathrm{pe}}$ \\
\hline 6 & $4.000 \mathrm{e}-3$ & 0.7158 & 0.0403 \\
8 & $7.986 \mathrm{e}-4$ & 0.9018 & 0.0395 \\
10 & $1.495 \mathrm{e}-4$ & 0.9849 & 0.0382 \\
12 & $4.964 \mathrm{e}-5$ & 0.9972 & 0.0342 \\
14 & 0 & 1 & 0.0381 \\
\hline
\end{tabular}

TABLE I

HMP-BASED SPECTRUM SENSING PERFORMANCE:

$P_{\mathrm{fa}}=$ FALSE ALARM PROBABILITY, $P_{\mathrm{d}}=$ DETECTION PROBABILITY, AND $P_{\mathrm{pe}}=$ PREDICTION ERROR PROBABILITY.

\begin{tabular}{|c|c|c|}
\hline SNR $(\mathrm{dB})$ & $P_{\mathrm{fa}}$ & $P_{\mathrm{d}}$ \\
\hline 6 & $4.000 \mathrm{e}-3$ & 0.6915 \\
8 & $7.986 \mathrm{e}-4$ & 0.8910 \\
10 & $1.495 \mathrm{e}-4$ & 0.9812 \\
12 & $4.964 \mathrm{e}-5$ & 0.9972 \\
14 & 0 & 1 \\
\hline
\end{tabular}

TABLE II

Energy Detector Performance: $P_{\mathrm{fa}}=$ FAlse alarm Probability, $P_{\mathrm{d}}=$ DETECTION PROBABILITY.

in Step 1). Note that a false alarm event occurs when the idle channel is detected as busy, and a missed detection event occurs when the busy channel is detected as idle.

6) Compute the probability of prediction error, $P_{\text {pe }}$, by comparing the predicted state sequence in Step 4) with the state sequence $\tilde{s}^{T}$ generated in Step 1).

Table I shows the values of $P_{\mathrm{fa}}, P_{\mathrm{d}}$, and $P_{\mathrm{pe}}$, respectively, for various SNR values. For this channel, the probability of false alarm $P_{f a}$ is very small for all values of SNR and decreases as the SNR increases. The probability $P_{\mathrm{d}}$ increases as the SNR increases. $P_{\mathrm{fa}}$ and $P_{\mathrm{d}}$ approach to 0 and 1 , respectively, when $\mathrm{SNR}$ is greater than $14 \mathrm{~dB}$. Unlike $P_{\mathrm{fa}}$ and $P_{\mathrm{d}}$ which have either increase or decrease trend, the probability of prediction error $P_{\text {pe }}$ has negligible fluctuation around 0.038 for all SNR values.

The probability of prediction error is computed as follows:

$$
P_{\text {pe }}=P_{\text {pe|off }} P_{\text {off }}+P_{\text {pe|on }} P_{\text {on }}
$$

where $P_{\mathrm{pe} \mid \text { off }}$ and $P_{\mathrm{pe} \mid \mathrm{on}}$ are the conditional probabilities of prediction error given channel off state and channel on state; $P_{\text {off }}$ and $P_{\text {on }}$ are the probabilities of off state and on state, respectively. Due to the measurement characteristics of the selected channel, the off probability $P_{\text {off }}$ is much larger than the on probability $P_{\text {on }}$. Consequently, the prediction error probability in the off state, $P_{\text {peloff }}$, is much smaller than the prediction error probability in the on state, $P_{\text {pe|on }}$. The overarll probability of prediction error shown in Table $I$ is small for most SNR levels because both $P_{\text {pe|off }}$ and the probability of the on state $P_{\text {on }}$ are small.

The HMP-based spectrum sensing algorithm was compared with an energy detector. The receiver operating characteristic (ROC) curve of the energy detector is shown in Fig. 4 for the various SNR values considered here. Table II shows the results for false alarm probability $P_{\mathrm{fa}}$ and detection probability $P_{\mathrm{d}}$. Comparing the two approaches shows that the HMPbased approach outperforms the energy detector for small SNR 


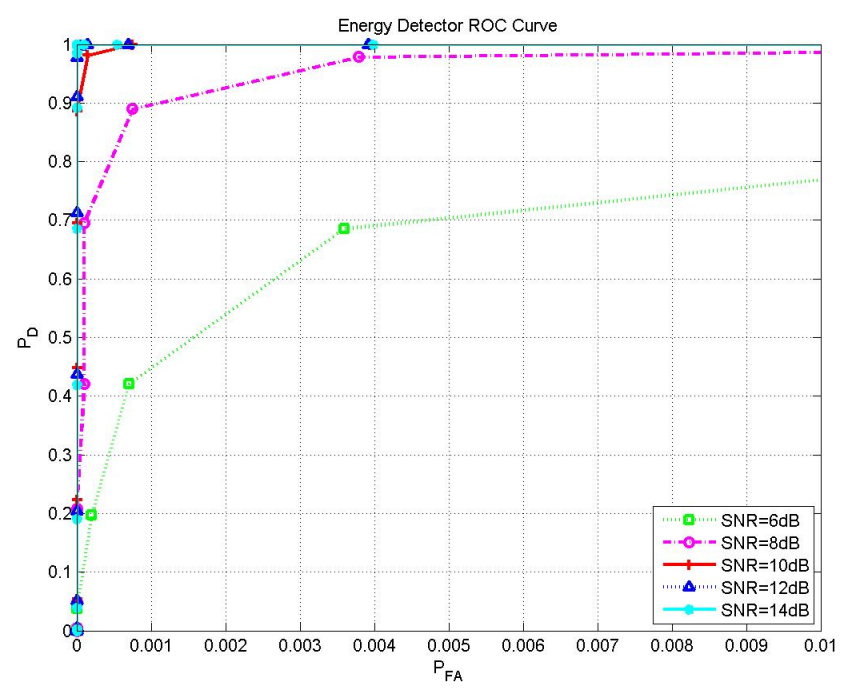

Fig. 4. Energy detector ROC curve.

values, i.e., when $\mathrm{SNR} \leq 10 \mathrm{~dB}$, and both detectors have comparable performance for higher SNR.

\section{CONCLUSION}

In this paper, we presented a method for dynamic spectrum access based on a hidden Markov process (HMP) model of a cognitive radio channel. The HMP has a continuous observation alphabet while the underlying state represents the on/off status of the primary user on a given channel. We evaluated the performance of the HMP-based spectrum sensing approach using real spectrum occupancy measurements.

The proposed HMP-based detector outperforms the commonly used energy detector especially in the regime of low signal-to-noise ratio and is more robust since it takes into account the measurement history. Another key feature of the HMP-based spectrum sensing is that it provides the predicted channel state with low probability of error while conventional detectors (e.g., energy detector) are not able to predict the future state. In ongoing work, we are investigating variations of the HMP model to obtain better prediction performance and to apply the results to the channel access policy. We are also investigating extensions of the proposed HMP-based approach to wideband spectrum sensing [12] and to scenarios involving collaboration among multiple cognitive radios distributed over a coverage area [13], [14].

\section{REFERENCES}

[1] Shared Spectrum Company, "General Survey of Radio Frequency Bands: $30 \mathrm{MHz}$ to $3 \mathrm{GHz}$," Shared Spectrum Company, Tech. Rep., Aug. 2010.

[2] Y. Ephraim and N. Merhav, "Hidden Markov processes," IEEE Trans. Information Theory, vol. 48, no. 6, June 2002.

[3] C. Ghosh and D. P. Agrawal, "Markov chain existence and hidden Markov models in spectrum sensing," Proc. IEEE PerCom'09, pp. 2-6, 2009.

[4] Z. Chen, Z. Hu, and R. C. Qiu, "Quickest spectrum detection using hidden Markov model for cognitive radio," in Proc. IEEE MILCOM'09, 2009.
[5] Z. Chen and R. C. Qiu, "Prediction of channel state for cognitive radio using higher-order hidden Markov model," March 2010, pp. 276 - 282.

[6] I. Akbar and W. Tranter, "Dynamic spectrum allocation in cognitive radio using hidden Markov models in spectrum sensing," in Proc. IEEE SoutheastCon'07, 2007, pp. 196-201.

[7] K. Kim, I. A. Akbar, K. K. Bae, J.-S. Um, C. M. Spooner, and J. H. Reed, "Cyclostationary approaches to signal detection and classification in cognitive radio," Proc. IEEE DySPAN'07, pp. 212-215, 2007.

[8] Y. Ephraim, D. Malah, and B.-H. Juang, "On the application of hidden Markov models for enhancing noisy speech," IEEE Trans. Acoustics Speech and Signal Processing, vol. 37, no. 12, Dec. 1989.

[9] D. R. Brillinger, Time Series-Data Analysis and Theory. New York: Holt, Rinehart \& Winston, 1975.

[10] T. Clancy and B. Walker, "Predictive dynamic spectrum access," Proc. SDR Forum Technical Conference, 2006.

[11] Z. Chen and R. C. Qiu, "Prediction of channel state for cognitive radio using higher-order hidden markov model," Proc of IEEE SoutheastCon, March 2010.

[12] Z. Quan, S. Cui, A. H. Sayed, and H. V. Poor, "Optimal multiband joint detection for spectrum sensing in cognitive radio networks," IEEE Trans. Signal Process., vol. 57, no. 3, pp. 1128-1140, Mar. 2009.

[13] J. Unnikrishnan and V. Veeravalli, "Cooperative sensing for primary detection in cognitive radio," pp. 18-27, Feb. 2008.

[14] T. Do and B. L. Mark, "Joint spatial-temporal spectrum sensing for cognitive radio networks," IEEE Trans. Vehicular Technology, vol. 59, no. 7 , pp. 3480-3490, Sept. 2010. 